Article

\title{
Feasibility Study of a Standardized Novel Animal Model for Cervical Vertebral Augmentation in Sheep Using a PTH Derivate Bioactive Material
}

\author{
Karina Klein ${ }^{1,2, *}$, Jason Schense ${ }^{3}$, Peter W. Kronen ${ }^{1,4,5}$, Nathalie Fouche ${ }^{1}$, Mariano Makara ${ }^{6}$,
} Katharina Kämpf ${ }^{1}$, Thomas Steffen ${ }^{7}$ and Brigitte von Rechenberg ${ }^{1,4}$

1 Musculoskeletal Research Unit (MSRU), Equine Department, University of Zurich, Winterthurerstrasse 260, CH-8057 Zurich, Switzerland; E-Mails: nathalie.fouche@web.de (N.F.); kkaempf@vetclinics.uzh.ch (K.K.); bvonrechenberg@vetclinics.uzh.ch (B.R.) Graduate School for Cellular and Biomedical Sciences, University of Bern, CH-3012 Bern, Switzerland

3 Kuros Biosurgery AG, Technoparkstrasse 1, CH-8005 Zurich, Switzerland; E-Mail: jason.schense@kuros.ch

4 Center for Applied Biotechnology and Molecular Medicine (CABMM), University of Zurich, Winterthurerstrasse 190, CH-8057 Zurich, Switzerland

5 Veterinary Anaesthesia Services - International (VAS), Zürcherstrasse 39, CH-8400 Winterthur, Switzerland; E-Mail: peter.kronen@vas-int.com

6 Division of Diagnostic Imaging, Vetsuisse Faculty, University of Zurich, Winterthurerstrasse 258c, CH-8057 Zurich, Switzerland; E-Mail: mariano.makara@gmail.com

7 Orthopaedic Research Laboratory, Department of Surgery, McGill University Hospital Center, 687 Pine Avenue West, Montreal, QC H3A 1A1, Canada; E-Mail: tsteffen@orl.mcgill.ca

* Author to whom correspondence should be addressed; E-Mail: kklein@vetclinics.uzh.ch; Tel.: +41-44-63-58-864.

Received: 29 May 2014; in revised form: 25 July 2014 / Accepted: 30 July 2014 / Published: 4 August 2014

Abstract: Prophylactic local treatment involving percutaneous vertebral augmentation using bioactive materials is a new treatment strategy in spine surgery in humans for vertebral bodies at risk. Standardized animal models for this procedure are almost non-existent. The purpose of this study was to: (i) prove the efficacy of PTH derivate bioactive materials for new bone formation; and (ii) create a new, highly standardized cervical vertebral augmentation model in sheep. Three different concentrations of a modified form of parathyroid hormone $(\mathrm{PTH})$ covalently bound to a fibrin matrix containing strontium carbonate were used. The same matrix without PTH and shams were used as controls. The bioactive materials were 
locally injected. Using a ventral surgical approach, a pre-set amount of material was injected under fluoroscopic guidance into the intertrabecular space of three vertebral bodies. Intravital fluorescent dyes were used to demonstrate new bone formation. After an observation period of four months, the animals were sacrificed, and vertebral bodies were processed for $\mu \mathrm{CT}$, histomorphometry, histology and sequential fluorescence evaluation. Enhanced localized bone activity and new bone formation in the injected area could be determined for all experimental groups in comparison to the matrix alone and sham with the highest values detected for the group with a medium concentration of PTH.

Keywords: vertebral augmentation; cervical spine; animal model; fibrin matrix; PTH derivate; sheep

\section{Introduction}

Symptomatic compression fractures of vertebral bodies occurring secondary to osteoporosis or neoplasia are a major public health problem in our aging society [1-5]. These fractures may cause persistent, often excruciating pain, spinal instability and kyphotic deformity, all of which potentially impair mobility and reduce the patient's quality of life [5-7]. Nowadays, percutaneous vertebroplasty (VP) is the treatment of choice to mechanically augment the spine. It is a successful, minimally invasive surgical procedure [8-10], which seems to be significantly better than non-surgical treatment in terms of pain relief, reduced hospital stay and recovery period [11]. The VP technique is performed through needle-injection of polymethylmethacrylate (PMMA) bone cement into a collapsed or weakened vertebra, this to stabilize, decompress or avoid further compression fractures (prophylactic VP). Common complications during the surgical procedure are bleeding at the puncture site, local infection and cement leakage into the spinal canal, the paravertebral tissues or the perivertebral venous system [12]. Cement extravasation, as well as bone marrow or fat cells forced out of the vertebra into the systemic circulation may lead to pulmonary embolism (PE), either without clinical signs or with severe cardiac distress symptoms, including death [5].

It is well known that PMMA increases the strength and stiffness of the injected vertebral bodies, but in the absence of osteoinductive properties and the inability to be degraded and replaced by new bone, it will remain just a placeholder. It may also lead to long-term complications, such as damage to neurons, toxicity or fracture of adjacent vertebral bodies $[3,13]$. Therefore, the search for new, preferably resorbable biomaterials, containing locally-released bioactive agents with osteoinductive properties to strengthen and regenerate weakened vertebral bodies is ongoing. Especially adjacent to the index level that was augmented with PMMA for immediate stability, enhancing local bone formation may in the future avoid in systemically-ill osteoporotic patients a domino effect of adjacent vertebral compression fractures [14].

A truncated form of the human parathyroid hormone $\left(\mathrm{PTH}_{1-34}\right)$ is already used as a registered pharmaceutical product for osteoporosis treatment. Intermittent systemic injections of $\mathrm{PTH}_{1-34}$ have shown anabolic effects on cancellous bone, resulting in increased bone mineral density [15]. PTH is able to bind directly to a cell surface receptor on osteoblasts, regulating the production of various 
cytokines (insulin-like growth factor 1 , transforming growth factor $\beta 1$, interleukin 1 (IL-1) and IL-6) and, thus, represents a strong anabolic agent for osteoblast precursors and an indirect anabolic factor for osteoclasts (IL-1 and IL-6) [15-17]. It regulates the balance between osteoblasts and osteoclasts, as well, thereby exerting a direct effect on bone turnover [15], and plays a primary role in calcium homeostasis [18]. In contrast to systemic use, a novel recent strategy is to use an engineered peptide variant of the active fragment of parathyroid hormone $\left(\mathrm{PTH}_{1-34}\right)$ that is enzymatically incorporated within a fibrin matrix via the coagulation transglutaminase (TG) factor XIIIa to translate the systemically used molecule into a local bone-inducing therapeutic agent [19]. The incorporation of a plasmin substrate sequence linker ( $\mathrm{pl}$ ) enables the cleavage of the peptide by endogenous plasmin. Via cell migration and infiltration, the peptide is activated and released only into the surrounding tissue for the local increase of bone formation. In previous in vivo studies using a tibia and femur augmentation model in sheep, various concentrations of this engineered parathyroid hormone $\left(\mathrm{TGplPTH}_{1-34}\right)$, incorporated in different formulations of fibrin matrices, has already shown good results [20]. However, it was unknown whether these results could actually be reproduced in the vertebral bodies, this due to its different anatomical structure, including high vascularity and sinus formations.

Safety aspects concerning the augmentation procedure must be considered, such that good visibility of the material during injection is key to avoid leakage into the spinal canal and damage to the spinal cord. Furthermore, the rheological properties of the material during injection are critical to avoid leakage into the vascular system, possibly causing pulmonary embolism or death. The radiopaque inorganic salt powder strontium carbonate has proven to be a good contrast agent. Strontium is also known to have positive effects on bone formation and for its capability to decrease bone resorption [21,22]. Different studies have shown that strontium could induce the replication of osteoblasts [23] and, concurrently, the apoptosis of osteoclasts [24] using a pathway involving the extracellular calcium-sensing receptor $(\mathrm{CaR})$. An in vivo study of Yang et al. using a calvarial defect model in rats showed that locally-delivered strontium was able to act as a bone anabolic agent and could activate the Wnt/ $\beta$-catenin signaling pathway, resulting in an accumulation of extracellular matrix, as well as enhanced osteogenesis and bone remodeling [25].

Good animal models for vertebral augmentation are rare. The sheep has been used as an experimental animal model for augmentation in the lumbar spine, although mainly for studying acute cardiovascular changes and embolization with immediate sacrifice on the table after augmentation [26-30]. Due to the different morphology of ovine vertebral bodies with a more trapezoid and slim vertebral body format compared to a round, solid and almost rectangular human vertebral body, a transpedicular approach for vertebral augmentation is highly hazardous and difficult to standardize in the lumbar area in sheep. Therefore, a new, highly standardized augmentation model in the cervical spine of sheep was developed for these experiments, where a ventral approach and customized instrumentation guaranteed the high reproducibility of the augmentation procedure. Apart from the safe deposition of the augmentation material, this model also involved a risk of pulmonary embolism similar to that found with human vertebral augmentation. Therefore, the study results may be better transferable to the human vertebral bone structure.

The aim of this study was to: (i) investigate the scaled effects of PTH derivate bioactive material as an enhancer for new bone formation in sheep; and (ii) create a novel, highly standardized animal model for vertebral augmentation in sheep. To reach aim (i), different concentrations of $\mathrm{TGplPTH}_{1-34}$, incorporated in the best performing fibrin matrix formulation from previous studies, were used as 
bioenhancers. For aim (2), cervical vertebrae in sheep were targeted focusing on the reproducibility and standardization of the model, including the surgical technique and overall evaluation. To the knowledge of the authors, this is the first report for both using the local application of an engineered peptide variant $\left(\mathrm{TGplPTH}_{1-34}\right)$ as a bioactive agent and reporting a standardized animal model in the cervical spine of sheep.

\section{Material and Methods}

\subsection{Study Design and Experimental Animals}

Seventeen adult female Swiss alpine sheep with a mean age of 3.2 years (range from 2 to 5 years) and a mean weight of $72.6 \mathrm{~kg}$ (54-89 kg) were used for this study, conducted adhering to the Swiss regulations of Animal Welfare (TSchG 455), with a specific permission granted by the federal authorities (Application No. 151/2009).

Using a cervical vertebral augmentation model in sheep, three different concentrations of a modified form of parathyroid hormone $\left(\mathrm{TGplPTH}_{1-34}\right)$ covalently bound to a fibrin matrix containing strontium carbonate, the same matrix without any $\mathrm{TGplPTH}_{1-34}$, as well as sham controls were tested and compared. Three vertebrae per sheep were augmented. Each group consisted of three sheep, with the exception of Group 2, where two sheep died during surgery, and therefore, two additional sheep were used (for the group distribution, see Table 1).

Table 1. Group distribution and clinical signs of pulmonary embolism. PTH, parathyroid hormone; PE, pulmonary embolism.

\begin{tabular}{|l|l|l|l|l|}
\hline Group & Material & $\begin{array}{l}\text { Number of } \\
\text { animals }\end{array}$ & $\begin{array}{l}\text { Number of } \\
\text { evaluated vertebra }\end{array}$ & $\begin{array}{l}\text { Clinical signs of pulmonary } \\
\text { embolism }\end{array}$ \\
\hline Matrix alone & FS+SrCO3 & 3 & 8 & $2 \times$ mild clinical signs \\
\hline Low & FS+SrCO3+PTH low conc. & 5 & 9 & $\begin{array}{l}2 \times \text { severe clinical signs led to death, } \\
1 \times \text { mild clinical signs }\end{array}$ \\
\hline Medium & FS+SrCO3+PTH low conc. & 3 & 8 & $1 \times$ mild clinical signs \\
\hline High & FS+SrCO3+PTH low conc. & 3 & 9 & $1 \times$ mild clinical signs \\
\hline Sham & No materials & 3 & 9 & \\
\hline
\end{tabular}

FS: Fibrin scaffold; SrCO3: Strontium carbonate; PTH low-high: TGplPTH1-34 concentration low-high;

Mild clinical signs of PE: intermittend bradycardia, Hypercapnia and mild oxygen desaturation

Using a ventral surgical approach and a customized aiming device, a fixed amount of biomaterial per vertebra (1-1.5 mL) was injected under fluoroscopic guidance in a standardized trajectory and to a standardized depth into the intertrabecular space of three vertebral bodies per sheep (between C2-C6). For the sham controls, the same procedure was performed without material injection. To detect pulmonary emboli, non-invasive airway dead space measurements were performed during surgery, as well as CT angiography of the thorax in all sheep prior to and after surgery [31]. After 16 weeks of follow-up, the sheep were sacrificed, and the harvested vertebral bodies were further processed for macroscopic assessment (signs of inflammation and irritation), $\mu \mathrm{CT}$ analysis and histology, including histomorphometry and evaluation of fluorescent dye incorporation. 


\subsection{Test Material}

Three different concentrations (low, medium, high) of a modified inactivated form of $\mathrm{PTH}_{1-34}$ (TGplPTH ${ }_{1-34}$ ) with a transglutaminase hook (TG-Hook) were evaluated (pending patent application) [19]. Using the TG-hook, the peptide was covalently bound and immobilized onto a fibrin matrix during the coagulation process and later locally released in vivo and activated by plasmin-induced proteolytic cleavage (via a plasmin substrate sequence linker (pl)) after cellular infiltration and replacement of the fibrin matrix with a newly secreted extra-cellular matrix. As a contrast agent for better radio-opacity and to improve the rheological properties during the injection procedure, $40 \%$ weight volume $(\mathrm{w} / \mathrm{v})$ strontium carbonate was added to the formulation. In one of the test groups, only the fibrin matrix including the strontium carbonate was used without $\mathrm{PTH}_{1-34}$.

\subsection{Instruments/Equipment}

In addition to the routine surgical instruments, a customized aiming device was developed to guarantee the reproducibility of surgical access and to standardize the access point on the bone surface for injection into the trabecular bone of the vertebral body (Figure 1a-c). Using a ventral surgical approach, the aiming device allows an exact drilling and subsequent positioning of the injection needle. This can be achieved via a central drill guide that is positioned on the promontorium of the caudal aspect of the vertebral body (Figure 1a), an aiming targeting tip lined up with the vertebra midline and two sharp long fixation dents (beside the midline) resting on the cranial deeper part of the vertebral body. An oval hole allows monitoring the drill guide tip placed on the promontorium while the drill guide remains positioned (Figure 1b).

Figure 1. This figure shows the aiming device in a correct position using a ventral approach to the vertebral body. (a) The lateral view represents the central drill guide in the correct position on the promontorium of the caudal aspect of the vertebral body. (b) The craniocaudal view shows the drill guide positioned on the promontorium, with an aiming targeting tip lined up with the vertebra midline and two sharp long dents, arranged symmetrically lateral of the midline, resting on the cranial deeper part of the vertebral body. In (c), the aiming device is shown as a whole with the handle, central drill guide and fixation dents.

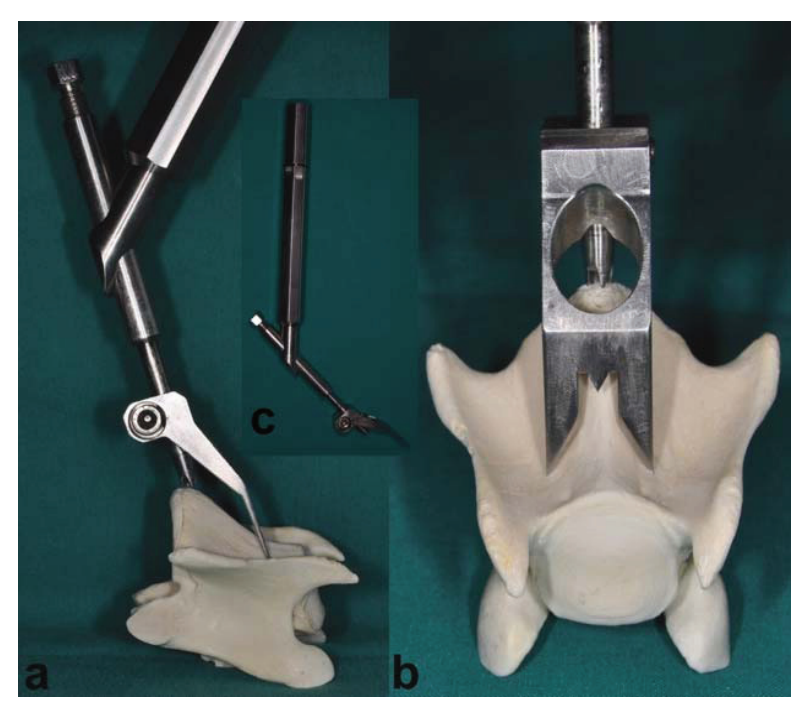


For injection of augmentation material, a $10 \mathrm{G}$ (3.2-mm diameter) $100 \mathrm{~mm}$-long special vertebroplasty needle (1394-1010, Optimed Medizinische Instrumente GmbH, Ettlingen, Germany) (Figure 2b) and an Optimed CementoRE gun (1392-0000, Optimed Medizinische Instrumente GmbH, Ettlingen, Germany) were used (Figure 2c).

Figure 2. The images (a-e) show the different steps of the surgical procedure: (a) an aiming device was used for repeatable and standardized drilling; (b) the vertebroplasty needle was correctly positioned; (c) material injection was performed in multiple steps using the Optimed CementoRE gun. Fluoroscopic imaging was used for repeatable and standardized drilling (d) and to detect material leakage during injection, as seen in (e), where leakage into vertebral vessels was detected at the caudal part of the two injected vertebrae (see the $\rightarrow$ ).

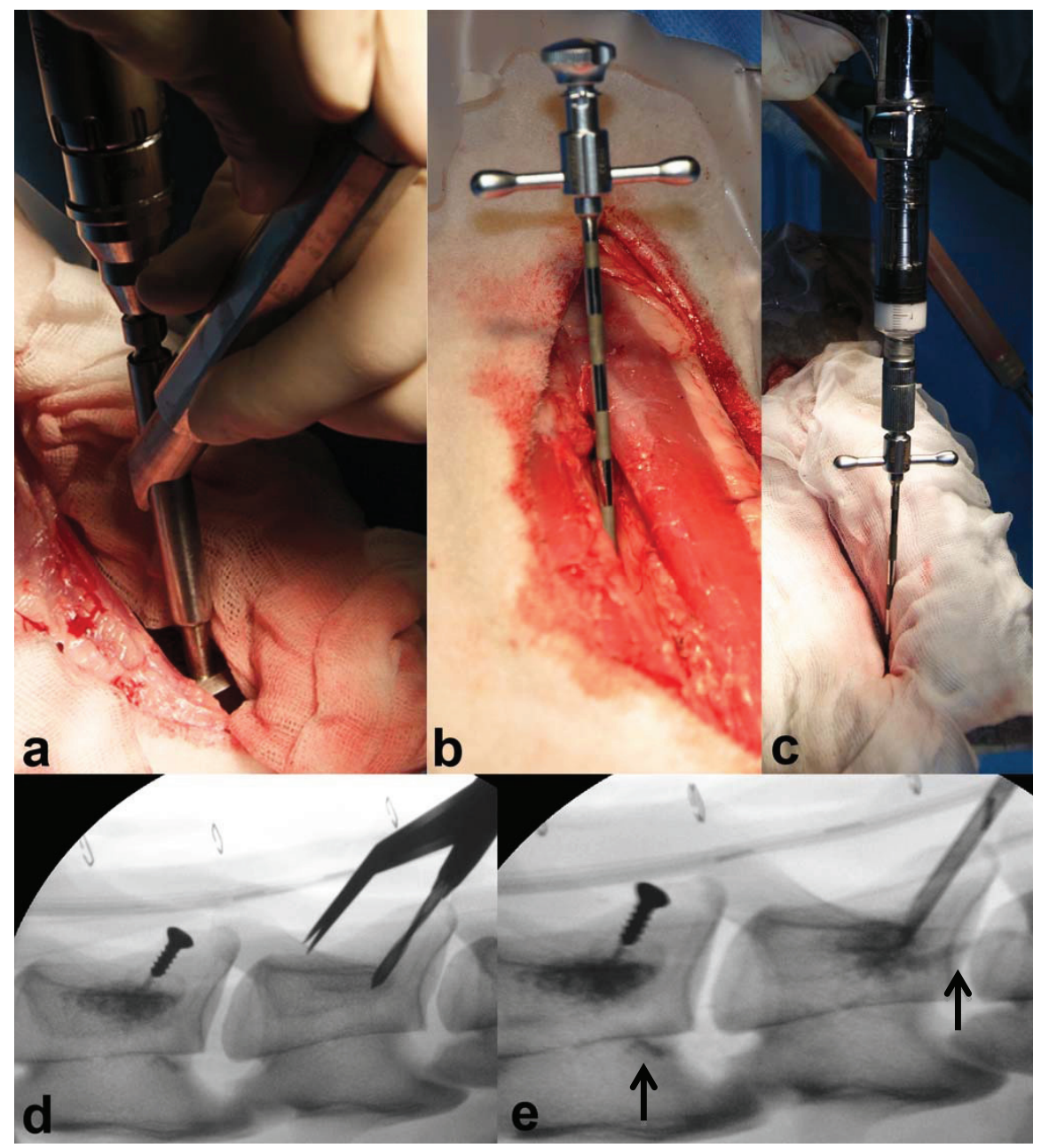

\subsection{Anesthesia}

A standard anesthetic protocol was used. After 24 hours of fasting and 30 minutes before induction of anesthesia, the animals were premedicated with buprenorphine (Temgesic ${ }^{\circledR}, 0.01 \mathrm{mg} / \mathrm{kg}$ i.m., Essex Chemie AG, Lucerne, Switzerland) and xylazine (Rompun ${ }^{\circledR} 2 \%, 0.1 \mathrm{mg} / \mathrm{kg}$ i.m., Streuli Pharma, Uznach, Switzerland). A catheter was placed into the vena cephalica antebrachii of one of the front legs and prophylactic antibiotics (Penicillin Natrium ${ }^{\circledR}$ 35,000 IU/kg i.v., G. Streuli Pharma, Uznach, 
Switzerland; gentamicin, Vetagent ${ }^{\circledR}, 4 \mathrm{mg} / \mathrm{kg}$ i.v., Veterinaria AG, Zurich, Switzerland), as well as a pre-emptive analgesic drug, carprofen (Rimadyl ${ }^{\circledR}, 4 \mathrm{mg} / \mathrm{kg}$ i.v., Pfizer SA, Zurich, Switzerland), were given intravenously. A booster for tetanus (Tetanus Serum Intervet, 3,000 IU/sheep s.c., Veterinaria AG Zurich, Switzerland) was administered subcutaneously.

Anesthesia was induced with diazepam (Valium ${ }^{\circledR}, 0.1 \mathrm{mg} / \mathrm{kg}$ i.v., Roche Pharma AG, Rheinach, Switzerland), ketamine (Narketan 10 ${ }^{\circledR}, 3 \mathrm{mg} / \mathrm{kg}$ i.v., Vetoquinol AG, Belp-Bern, Switzerland) and propofol (Propofol ${ }^{\circledR}, 0.4-2 \mathrm{mg} / \mathrm{kg}$ i.v., Fresenius Kabi, Stans, Switzerland), the latter administered to effect. After laryngeal desensitization with lidocaine spray (xylocaine spray $10 \%, 3$ pumps of $0.1 \mathrm{~mL}$ each, Astra Zeneca AG, Zug, Switzerland), the trachea was intubated and correct placement was confirmed by expired carbon dioxide monitoring $\left(\mathrm{F}_{\mathrm{et}} \mathrm{CO}_{2}\right)$. Anesthesia was maintained with a balanced anesthetic protocol employing the administration of isoflurane $(1 \%-2 \%)$ in oxygen via an adult F-circuit (circle system, Intersurgical, Berkshire, U.K.), a variable rate infusion of propofol (Propofol ${ }^{\circledR}$, 0.5-1 mg/kg/h, Fresenius Kabi, Stans, Switzerland), as well as lidocaine given at the beginning of the surgical procedure as bolus $\left(1 \mathrm{mg} / \mathrm{kg}\right.$ ) and afterwards as constant rate infusion (Lidocaine ${ }^{\circledR}$, $40 \mu \mathrm{g} / \mathrm{kg} / \mathrm{min}$, Kantonsapotheke, Zurich, Switzerland).

Monitoring parameters included electrocardiogram (ECG), heart rate, pulse rate and invasively measured blood pressures (systolic, mean and diastolic arterial) via an arterial catheter in an auricular artery. Furthermore, inspired and expired concentrations of carbon dioxide, oxygen and isoflurane, as well as esophageal temperature and saturation of arterial blood $\left(\mathrm{SpO}_{2}\right)$ were monitored. All parameters were constantly measured and recorded in 10-minute intervals. Intraoperatively, fluids Ringer's lactate solution (Ringer Lactat ${ }^{\circledR}$, Bichsel, Interlaken, Switzerland) was administered at a rate of $10 \mathrm{~mL} / \mathrm{kg} / \mathrm{h}$, and heat was applied using a forced-air warming device (Bair Hugger ${ }^{\circledR}$ 505, Augustine Healthcare, Eden Prairie, MN, USA).

Intermittent positive pressure ventilation was applied (if needed) to maintain normocapnia. A Swan-Ganz-catheter was inserted via the jugular vein into the pulmonary artery to allow invasive measurements of pulmonary artery pressure and cardiac output. Physiologic airway dead space measurements were performed with the non-invasive cardiac output monitor, NICO ${ }^{\circledR}$ (Respironics Novametrix, Inc., Wallingford, CT, USA). The results of this comparison between invasive and non-invasive measurements are not included in this paper and will be described elsewhere.

\subsection{Surgical Procedure}

The anesthetized sheep were placed in dorsal recumbency with the forelimbs retracted caudally and fixed to the surgery table. The neck was bent slightly upwards for an easier approach to the vertebral bodies. The correct position of the sheep was further supported with an inflatable surgery mat, rubber foam rolls and wedges. To prevent bloating and regurgitation, a stomach tube was placed prior to surgery. The tube was maintained throughout the entire surgery until recovery in sternal recumbency. The ventral aspect of the neck was shaved and surgically scrubbed and cleansed. The animals were draped according to routine. A midline skin incision was performed from the pharyngeal region extending caudally to the level of the thoracic inlet. The subcutis and superficial facial planes were bluntly dissected to expose sternohyoid and pharyngeal muscles. A blunt and careful dissection was made in the midline in between the left and right sternohyoid muscles. The jugular vein, carotid artery, 
vagal nerve, trachea and oesophagus were carefully retracted using a blunt retractor wrapped in gauze. Exposure of the ventral aspect of the vertebral bodies was maintained by securing the retractors in place. The desired vertebra was identified by palpating the transverse processes and the intervertebral spaces. The identification of the area of interest was performed using two anatomical landmarks: the promontorium caudal to the desired vertebral body and the deeper laying cranial aspect of the vertebra. The muscles covering the caudal aspect of the vertebral body were bluntly divided to expose the area of interest. Hemostasis was meticulously kept using electrocautery. The aiming device was placed with the tip of the drill guide at the promontorium in the cranial drill direction (Figure 1a-c).

Under fluoroscopic control, the correct placement of the aiming device and subsequent needle positioning was validated to avoid inaccurate injections. A drill hole with a diameter of $2.8 \mathrm{~mm}$ was created in the vertebral body (Figure $2 \mathrm{a}$ ) followed by placing the special vertebroplasty needle using a Kirschner wire as guide for the correct direction of the needle path. Afterwards, the needle was pushed forward, till its tip was firmly seated and surrounded by bone (Figure 2b).

Material injections were done in multiple steps under fluoroscopic guidance (Figure 2e). Needle filling with augmentation material was done slowly with the aid of the Optimed CementoRE gun (Figure 2c). Subsequently, the gun was removed, and the stylet was used to push the material into the bone, so as to provide exact quantities of injectate. The filling-clearing procedure was repeated one or two times, until a target between 1.5 and $1.8 \mathrm{~mL}$ was injected within the intertrabecular space. Using fluoroscopy, the leakage of material into a vessel and thrombus formation was recorded, the injection needle slightly advanced and injection continued. At completion of the procedure, the vertebroplasty needle was removed and the insertion point closed with a 3.5- $\mathrm{mm}$ diameter self-tapping screw (8 $\mathrm{mm}$ in length, Synthes, Oberdorf, Switzerland) (Figure 2d and 2e). The screw was used to later determine the direction of the needle and insertion point at the time of sacrifice.

Afterwards, the muscles were repositioned, and the closure of the soft tissue was routinely done using resorbable suture material (Vicryl ${ }^{\circledR}$ polyglactin, 2-0, Johnson \& Johnson Int., Brussels, Belgium) in a simple continuous fashion, while the skin was closed with a skin stapler (Appose ULV, United States Surgical). The Swan-Ganz catheter was removed, and a normal catheter (Vygonyle $S^{\circledR}$ G14, Vygon GmbH, Aachen, Germany) was placed in the other jugular vein. Intra-operative fluoroscopy images were taken in right lateral view throughout the surgery to document the surgical technique and the injection steps. Immediately after surgery, fluoroscopy images in latero-lateral and ventrodorsal view were taken to document the distribution of the injected material in two projection plans.

\subsection{Postoperative Treatment}

The animals were supervised during recovery from anesthesia until they could stand on their own, walk around and eat without further complications. They were kept in small groups, and their health status was checked twice daily. Antibiotic and analgesia administrations were continued for 4 days intravenously using the same dosage as perioperatively applied.

Additional analgesia treatment was provided to reduce postoperative pain using intramuscular injections of buprenorphine (Temgesic ${ }^{\circledR}, 0.01 \mathrm{mg} / \mathrm{kg}$, Essex Chemie AG, Luzern, Switzerland) every 4 hours for a total of three applications. 


\subsection{In Vivo Fluorescence Labeling}

Different fluorescence markers were subcutaneously injected at 6 weeks (calcein green, $5 \mathrm{mg} / \mathrm{kg}$ BW s.c., Fluka AG, Buchs, Switzerland), 12 weeks (xylenol orange, $90 \mathrm{mg} / \mathrm{kg} \mathrm{BW} \mathrm{s.c.,} \mathrm{Fluka} \mathrm{AG)} \mathrm{and}$ three days prior to sacrifice (oxytetracycline, $20 \mathrm{mg} / \mathrm{kg}$ BW s.c., Engemycin ${ }^{\circledR} 10 \%$, MSD Animal health $\mathrm{GmbH}$, Lucerne, Switzerland) to dynamically represent new bone formation and remodeling.

\subsection{Sacrifice and Specimen Preparation for Evaluative Procedures}

All animals were sacrificed after a follow up period of 16 weeks, and the three treated cervical vertebrae were harvested for final analysis. Augmentation sites were examined macroscopically, and digital photographs were taken to document tissue reaction at the surgical site (Nikon Digital Camera D5000, 2009 Nikon Corporation $^{\circledR}$ ). Radiographs were taken using a Faxitron (Faxitron X-Ray Systems, Hewlett Packard, Mc Minnville Division, OR, USA) to visualize the detailed bone structure and detect remaining augmentation material. Subsequently, each vertebra was cut in a smaller segment containing only the injected vertebral body for evaluative procedures. An additional radiograph was performed to qualitatively assess new bone formation in the vertebral bodies. The bone samples were fixed in $40 \%$ ethanol for one week, followed by a series of ethanol dehydration steps $(50 \%-100 \%)$ within 6 days. $\mu$-CT analysis of the specimen was performed in 70\% ethanol fixation (b-cube AG, Bio-Technopark, Schlieren-Zurich, Switzerland). After complete dehydration, the samples were degreased in xylene and subsequently infiltrated in liquid methymethacrylate (MMA) until polymerization occurred [20]. Using a special band saw $\left(\right.$ EXAKT $^{\circledR}$ Band System 300/301, EXAKT ${ }^{\circledR}$ Apparatebau $\mathrm{GmbH} \& \mathrm{Co} \mathrm{KG}$, Norderstedt, Germany), cutting of the ground sections (300 $\mu \mathrm{m})$ and native fluorescence sections $(350 \mu \mathrm{m})$ were performed. The sections were oriented along the original needle path in the midline of the vertebral body, with the needle path itself, the tip of the needle and the surrounding tissue defined as the region of interest. Before mounting (Cementit ${ }^{\circledR}$ CA 12, Merz + Benteli AG, Niederwangen, Switzerland) the sections on plastic slides (ACROPAL, Maagtechnic, Duebendorf, Switzerland), microradiographs were taken with the Faxitron $(27 \mathrm{kV}, 11 \mathrm{~s}$; Fuji Photo Film Co Ltd, Tokyo, Japan). Ground sections were polished (Exakt ${ }^{\circledR}$ Mikro- Schleifsystem 400CS, Exakt Apparatebau GmbH, Norderstedt, Germany) and surface stained with toluidine blue. The native sections for fluorescence were mounted on pellucid, acrylic Plexiglas slides (Maagtechnic, Duebendorf, Switzerland) and wrapped in aluminum foil to protect slides from bleaching. For thin section preparation, the remaining bone blocks were cut in smaller segments comprising only the tip of the needle and surrounding tissue. Polymerization was achieved in special customized Teflon forms (D. Nadler, JOSSI AG, Islikon, Switzerland) [20]. Afterwards, the small blocks were mounted on plastic frames and cut using a microtome (Leica ${ }^{\circledR}$ RM 2155, Leica Instruments GmbH). After mounting on glass slides and pressing for two days in the incubator, the sections were stained with either toluidine blue or van Kossa/McNeal. 


\subsection{Analysis}

\subsubsection{Micro-Computed Tomography Analysis}

Micro-computed tomography $(\mu \mathrm{CT})$ analysis of the vertebral bodies was performed to qualitatively assess the bone volume density and trabecular thickness, as well as the location and amount of residual augmentation material within the treated levels. The samples were scanned by a micro-computed tomography system ( $\mu \mathrm{CT}$ 40, Scanco Medical AG, Brüttisellen, Switzerland). The measured data were filtered and then segmented to separate the bone and the potentially present residual biomaterial from the background using a global thresholding procedure. For samples with identified residual biomaterial, a multi-level segmentation technique was used to segment bone and residual material in two separate phases, whereas for samples without residual material, a simple segmentation procedure was applied to separate bone from background. For all samples, the same thresholds were used. The measurements were performed using semiautomatic masks of nine overlapping hollow sphere shells in different sizes centered at the tip of the needle, used to calculate average bone volume density and trabecular thickness within each sphere shell (Figure 3).

Figure 3. This figure represents one slice through the nine hollow sphere shells used for the analysis. The needle path and the cortical bone areas were excluded from the analysis.

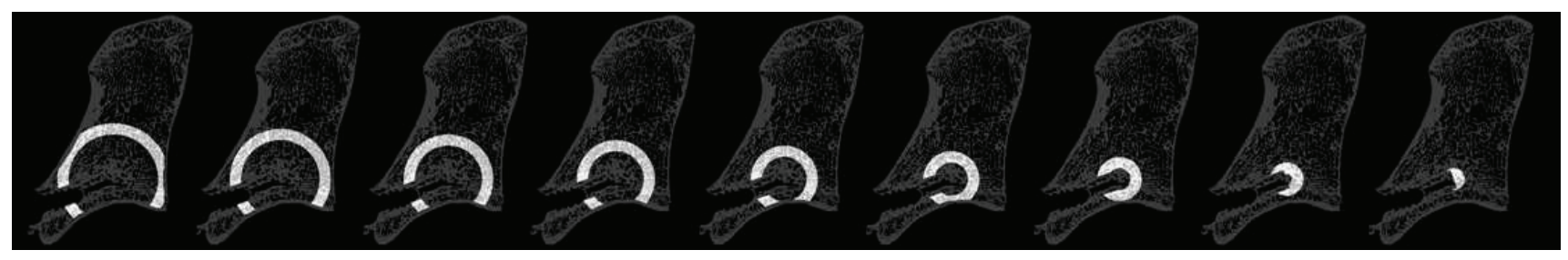

Figure 4. This figure shows the different masks created for density analysis. (A) The inner mask to exclude the cortical bone from the analysis. (B) The defined cylinder (Ø $3.5 \mathrm{~mm}$ ) representing the original needle path with two manually determined points "tip" and "base". This cylinder was subtracted from the hollow sphere shells. Furthermore, the part of the sphere shell not lying in the trabecular bone was subtracted from the sphere shell. (C) An example of the final sphere shell mask.
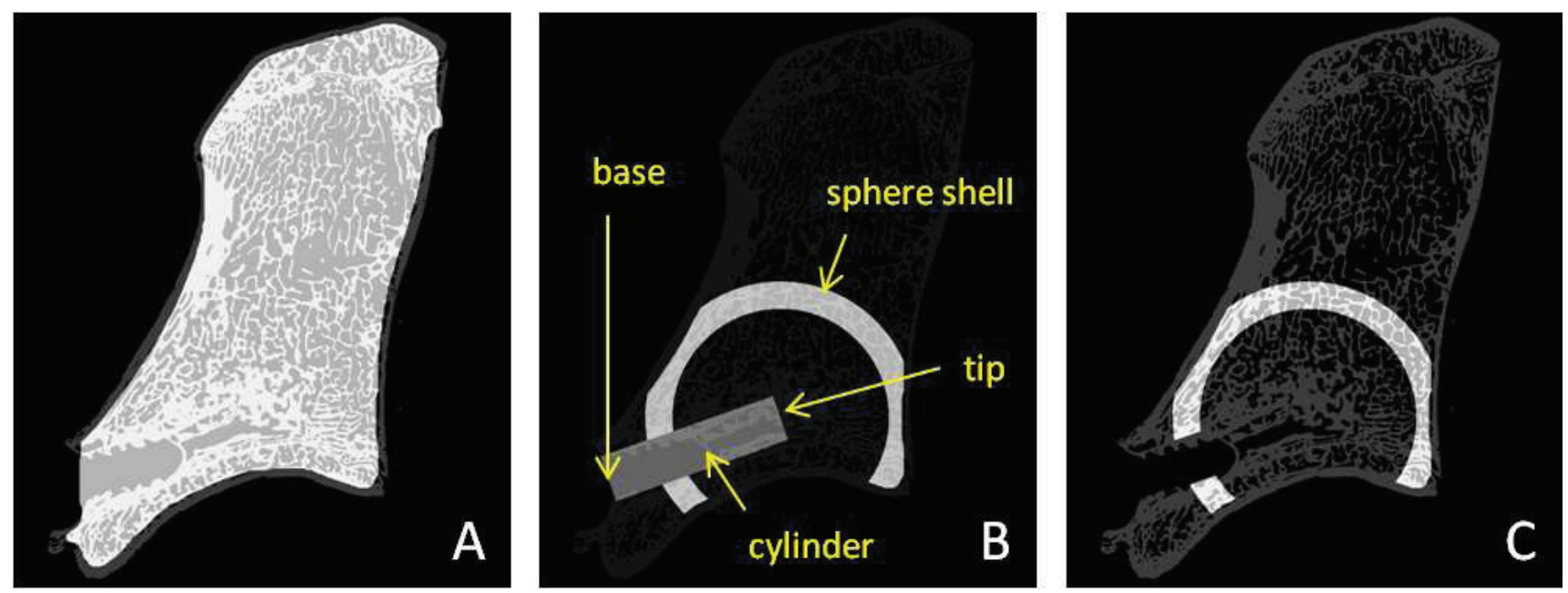
Using these sphere shells, the calculation of average material distribution and bone volume density in correlation to the radial distance from the tip of the needle was possible. To measure only the trabecular bone area, a region correlating to the cylindrical needle path and the cortical bone shell were excluded from the analysis. For the original needle path, a cylinder slightly larger than the original diameter of the needle was chosen, in order to exclude the region of any bone debris produced during the needle insertion procedure (Figure 4).

Integrating the values of the spherical shell data, the bone volume density in full thickness spheres could be computed (Figure 5).

Figure 5. This figure shows one slice with 4 full thickness spheres with $8 \mathrm{~mm}, 12 \mathrm{~mm}$, $16 \mathrm{~mm}$ and $20 \mathrm{~mm}$ diameter, respectively.

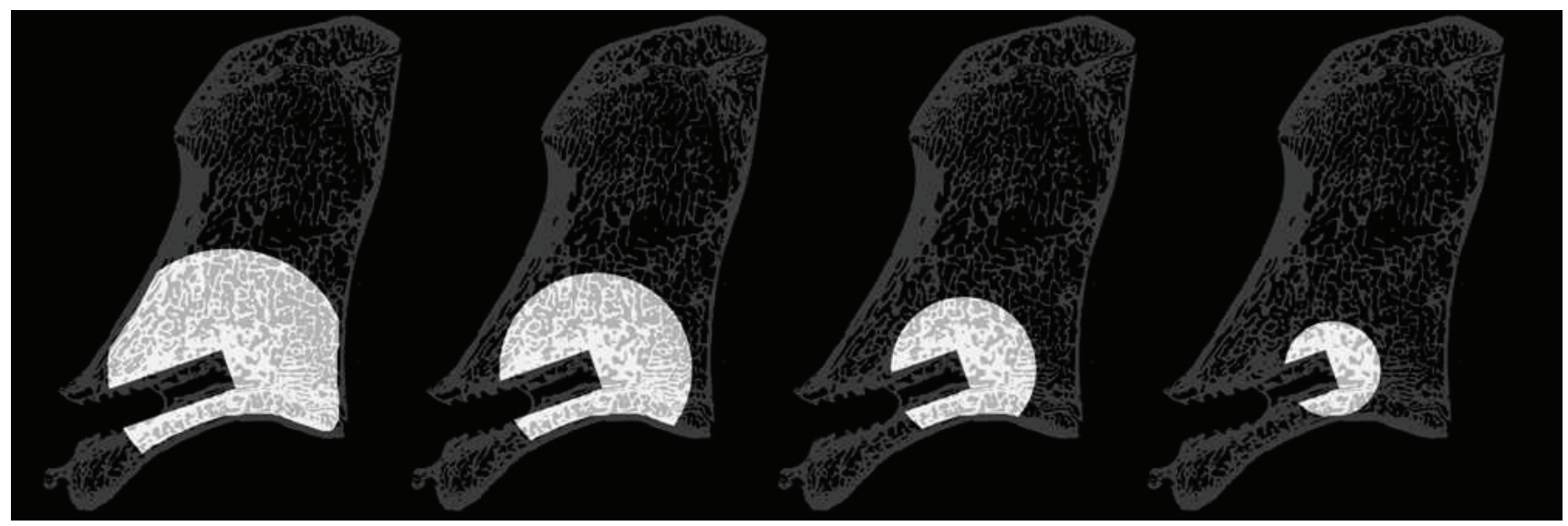

Figure 6. This figure shows a full sphere of $12 \mathrm{~mm}$ in diameter and a sphere shell with more than $12 \mathrm{~mm}$ in diameter. The inner 'Sphere12' is representative of the injected region of biomaterial, subsequently called 'augmented'. The outer 'Shell12+' is representative of the untreated bone region and used as internal sample reference, subsequently called 'unaugmented'. Needle path and cortical bone regions (grey shaded areas) were excluded from the analysis.

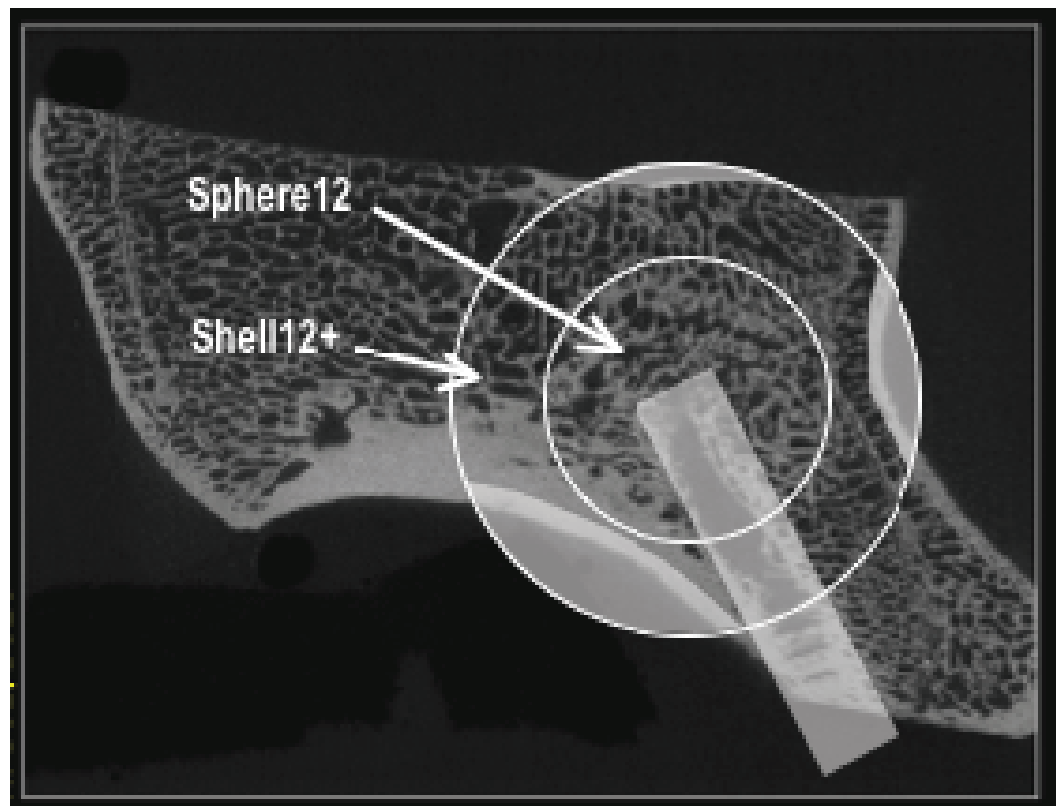


With the injected volume of approximately 1-1.5 $\mathrm{mL}$ material, the sum of sphere shells up to $12 \mathrm{~mm}$ in diameter were assumed as the injected area (consecutively called the full thickness sphere), whereas the sum of all larger sphere shells were assumed to be non-injected (Figure 6).

\subsubsection{Histological Evaluation}

Histomorphometry was performed using digitally recorded images of the ground sections (5.6-fold magnification, macroscope Leica Z6 APOA, Leica DFC 420C, Glattbrugg, Switzerland). For standardization of the evaluated area, three circles of $5 \mathrm{~mm}$ in diameter were chosen around the tip of the needle (Figure 7). The structures of interest within these circles were manually color-highlighted interactively using Adobe Photoshop Elements 10 (Adobe Systems, San Jose, CA, USA). Old bone was highlighted in blue (R: 0; G: 210; B: 255), newly formed bone in green (R: 34; G: 79; B: 7), granulation tissue in pink (R: 242; G: 40; B: 211) and residual augmentation material in beige (R: 198; G: 156; B: 96) using a standard pixel-detecting tool of Adobe Photoshop. Afterwards, the colored images were analyzed using a special image analysis software (QUIPS/QWIN, Leica standard, V.3.0, 2003, Leica-Microsystems, Heerbrugg, Switzerland), and the colored fractions were automatically detected and measured in pixels using binary segmentation of a standard macro-routine. Afterwards the pixels of each whole circle were set as $100 \%$ and the different colored fractions converted into percentages. Circles including the growth plate were excluded from the analysis, to avoid higher rates of new bone present independently of the applied material. Cortical bone in the circles was excluded from the region of interest and the rest of the circle considered as $100 \%$, since disproportional high-density old bone is present in the cortex. The average of all three circles per sample were used for statistical analysis. To reduce any inter-observer variability, only one examiner performed the analysis; certain randomly chosen images were evaluated twice as the control.

Figure 7. Example of a histology ground section for histomorphometric evaluation. Three circles $(\varnothing 5 \mathrm{~mm})$ at the tip of the needle were chosen for the evaluation of the percentage of old bone, newly formed bone, total bone and granulation tissue within the area of interest.

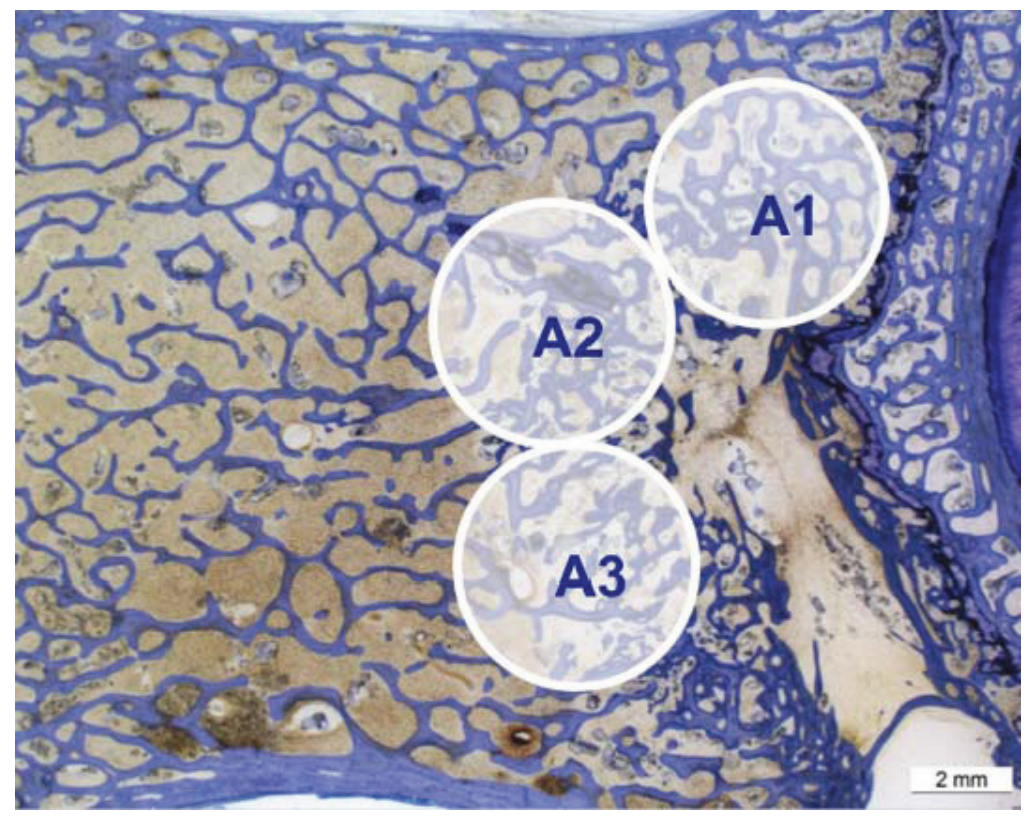


Thin sections were evaluated semi-quantitatively for cellular reactions using a microscope (Leica ${ }^{\circledR}$ DMR, Glattbrugg, Switzerland) in 10- to 20-fold magnification. Scores were given for presence of macrophages, mononuclear cells, osteoclasts, multinucleated foreign body cells, amount of osteoid seam, thickness of osteoid seam and amount of residual material (Table 2). Three power fields per section were assessed with two peripheral and one central in the injected area. The average of all three scoring areas for each augmentation site was statistically evaluated.

Table 2. Indicators for cellular reactions evaluated in the semi-quantitative histology scoring. Low scores indicate low numbers and high scores high numbers of cells, material or osteoid seams.

\begin{tabular}{|l|l|c|}
\hline Indicator & $\begin{array}{l}\text { Observable value } \\
\text { (in the circular scoring area) }\end{array}$ & $\begin{array}{l}\text { Scoring range } \\
\text { (for each single circular } \\
\text { scoring area) }\end{array}$ \\
\hline Mononuclear cells & $\begin{array}{l}\text { Percentage of mononuclear cells compared to the } \\
\text { overall number of cells }\end{array}$ & $0-3$ \\
\hline Macrophages & $\begin{array}{l}\text { Percentage of macrophages compared to the overall } \\
\text { number of cells }\end{array}$ & $0-3$ \\
\hline $\begin{array}{l}\text { Multinucleated foreign } \\
\text { body cells }\end{array}$ & Absolute number of multinucleated foreign body cells & $0-3$ \\
\hline Fibroblasts & $\begin{array}{l}\text { Percentage of fibroblast compared to the overall } \\
\text { number of cells }\end{array}$ & $0-3$ \\
\hline Osteoclasts & Absolute number of osteoclasts & $0-3$ \\
\hline Amount of osteoid seam & Percentage of bone surface covered by osteoid & $0-3$ \\
\hline Thickness of osteoid seam & $\begin{array}{l}\text { Thickness of the osteoid compared to the average size } \\
\text { of an osteoblast }\end{array}$ & $0-3$ \\
\hline Residual material & Percentage of surface occupied by residual material & $0-3$ \\
\hline
\end{tabular}

Fluorescent sections were evaluated semi-quantitatively for the differences of dye integration between groups and at different time points (calcein green at 6 weeks, xylenol orange at 12 weeks and oxytetracycline at 16 weeks post-surgery). Digital images of the region of interest at the tip of the needle were recorded. Therefore, 30 single images were taken in a 1.25 magnification and merged together using a special microscope, camera and specific merging software ("Stitching function"; Leica $^{\circledR}$ DM 600B, Leica ${ }^{\circledR}$ DFC 350 FX, Leica ${ }^{\circledR}$ Microsystems CMS GmbH, Mannheim, Germany). Scores were given for the percentage of each fluorescent dye in the region of interest (considered as $100 \%$ ) with $0 \%=0,1 \%-25 \%=1,26 \%-50 \%=2,>50 \%=3$.

\subsubsection{Statistical Analysis}

Results were analyzed statistically by means of PASWStatistics ${ }^{\circledR}$ software (Base for Mac OS X, Version 21.0, Chicago, IL, USA). Overall differences between groups were assessed using a factorial analysis of variance (ANOVA). Differences between individual groups were calculated with post hoc tests according to Scheffe. $p$-values $<0.05$ were considered statistically significant. 


\section{Results}

\subsection{Surgery, Postoperative Period and Sacrifice}

A total of 17 female adult sheep were treated with the cervical vertebral augmentation model. Out of these 17 animals, two (11.8\%) died during surgery after augmentation of the first vertebra. Death was attributed to severe cardiac distress, including a sudden decrease in arterial blood pressure and end-expired $\mathrm{CO}_{2}$ by approximately $50 \%$, followed by cardiac arrest. The other 15 sheep completed the 16-week follow-up period successfully. Out of these, 5/15 animals (29.4\%) developed mild symptoms of pulmonary embolism, but recovered while still in surgery without further complications. No significant difference between treatment groups for the incidence of pulmonary embolism was found. Although only seven animals showed clinical signs of pulmonary embolism, the leakage of biomaterial $(0.1-0.5 \mathrm{~mL})$ into the venous circulation was observed in 19/36 (52.7\%) material-treated augmentation sites (i.e., sham group excluded) using fluoroscopy during the injection procedure.

In total, 43/45 augmentation sites were treated. Using the aiming device and fluoroscopic imaging for standardized needle placement and material injection, the needle could be positioned successfully during the first attempt in 42/45 augmentation sites (three vertebrae per sheep, 15 remaining sheep). Only in 3/45 augmentation sites (medium and high concentration groups and sham group), needle positioning was unsuccessful in the first attempt. In these cases, the drill guide was repositioned and drilling was repeated due to a misalignment of the first drilling. In one of these injection sites (medium conc. group), no material could be injected in the vertebra, because the first drilling had perforated the opposite cortical bone with a disproportional high risk of leakage outside the vertebral body into the spinal canal. Another unplanned deviation of the surgical procedure occurred in the first sheep of the study, where only two vertebrae were treated due to a very long anesthesia and surgery period. For health and survival reasons, the surgery was stopped after the augmentation into the second vertebra.

Another minor surgical complication at one augmentation site was the breaking of the needle during the extraction procedure from the bone. The small broken part of the needle remained within the bone. However, no further clinical complications resulted from this event, and the augmentation site was evaluated according to the standard protocol.

Under fluoroscopic guidance during surgery, all formulations showed good radio-opacity and a sphere-like shape distribution around the tip of the needle with an injected amount between 1 and $1.5 \mathrm{~mL}$ of augmentation material.

The overall surgical procedure for each animal lasted between 45 and 60 minutes. After a short recovery period following anesthesia (approximately 30 minutes), the sheep roamed freely in the stables or on pasture without lameness or other visible discomfort. During the 16-week follow-up, no signs of wound infections or inflammation were noticed in any of the 15 remaining sheep.

The en-bloc harvesting process of the cervical spine, including all treated vertebra, was uneventful, and all samples could be prepared for further evaluation. By setting the screw for needle direction after sacrifice and using the customized aiming device during the surgical procedure, the central area of augmentation within the vertebral bodies could be found in all samples with a longitudinal cut through the original needle path. 


\subsection{Analysis}

\subsubsection{Micro-Computed Tomography}

Results of $\mu \mathrm{CT}$ are given in Table 3 . With the $\mu \mathrm{CT}$ images, the suitability of the surgical method could be confirmed with respect to a consistent, standardized needle placement in the desired and predicted trabecular bone space. For all treatment groups, a higher bone volume density and an increased trabecular thickness was found when compared to the sham group, indicating a bone enhancing effect of all material formulations (Table 3, Figure 8). Trabecular thickness was similar in the treated groups and the matrix alone group. This was combined with a radial distribution for both parameters with higher values central in the augmented area (until sphere 12) and decreasing values to the unaugmented area (sphere $>12$ ). Residual augmentation material was only detected in the matrix-only treatment group (no TGplPTH ${ }_{1-34}, 4 / 7$ samples) and the low concentration group (2/9 samples). No other treatment group had residual material detected.

Table 3. Means and SD of the morphometric $\mu \mathrm{CT}$ measurements for BV/TV (bone volume density) and Tb.Th. (trabecular thickness) for three spheres: $8 \mathrm{~mm}, 12 \mathrm{~mm}$ and $16 \mathrm{~mm}$. Note that the BV/TV in the larger $12 \mathrm{~mm}$ sphere is higher for the treated groups, while trabecular thickness is equal to the matrix alone group and only higher compared to the sham.

\begin{tabular}{|c|c|c|c|c|c|c|}
\hline Group & $\begin{array}{c}\text { BV/TV [\%] } \\
(\text { sphere } 8 \mathrm{~mm})\end{array}$ & $\begin{array}{c}\text { BV/TV [\%] } \\
(\text { sphere 12 } \mathrm{mm})\end{array}$ & $\begin{array}{c}\text { BV/TV [\%] } \\
(\text { sphere 16 mm) }\end{array}$ & $\begin{array}{c}\text { Tb.Th }[\mathbf{m m}] \\
(\text { sphere } 8 \mathrm{~mm})\end{array}$ & $\begin{array}{c}\text { Tb.Th }[\mathbf{m m}] \\
(\text { sphere 12 mm) }\end{array}$ & $\begin{array}{c}\text { Tb.Th }[\mathbf{m m}] \\
(\text { sphere 16 mm })\end{array}$ \\
\hline Matrix alone & $52.2 \pm 9.0$ & $44.7 \pm 11.0$ & $41.0 \pm 8.9$ & $0.43 \pm 0.07$ & $0.38 \pm 0.08$ & $0.34 \pm 0.06$ \\
\hline Low & $52.9 \pm 8.6$ & $47.3 \pm 6.0$ & $45.6 \pm 5.1$ & $0.34 \pm 0.07$ & $0.30 \pm 0.06$ & $0.31 \pm 0.08$ \\
\hline Medium & $61.5 \pm 3.8$ & $55.1 \pm 4.8$ & $50.3 \pm 4.7$ & $0.40 \pm 0.10$ & $0.37 \pm 0.08$ & $0.34 \pm 0.08$ \\
\hline High & $50.2 \pm 16.6$ & $46.7 \pm 15.5$ & $43.8 \pm 14.1$ & $0.34 \pm 0.11$ & $0.31 \pm 0.10$ & $0.28 \pm 0.08$ \\
\hline Sham & $43.6 \pm 10.3$ & $45.0 \pm 9.1$ & $46.5 \pm 8.6$ & $0.27 \pm 0.07$ & $0.27 \pm 0.07$ & $0.28 \pm 0.06$ \\
\hline
\end{tabular}

Figure 8. Three representative sagittal sections of the $\mu \mathrm{CT}$ scans: (a) a sample of the control group; (b) a sample of the group with medium $\mathrm{TGplPTH}_{1-34}$ concentration is illustrated; (c) a sample of the group with high $\mathrm{TGplPTH}_{1-34}$ concentration is shown. A denser bone area in the injected region could be distinguished for the samples in (b) and (c), where the augmentation material was injected. Increased trabecular thickness is visible in the circles of the outlined augmentation area.
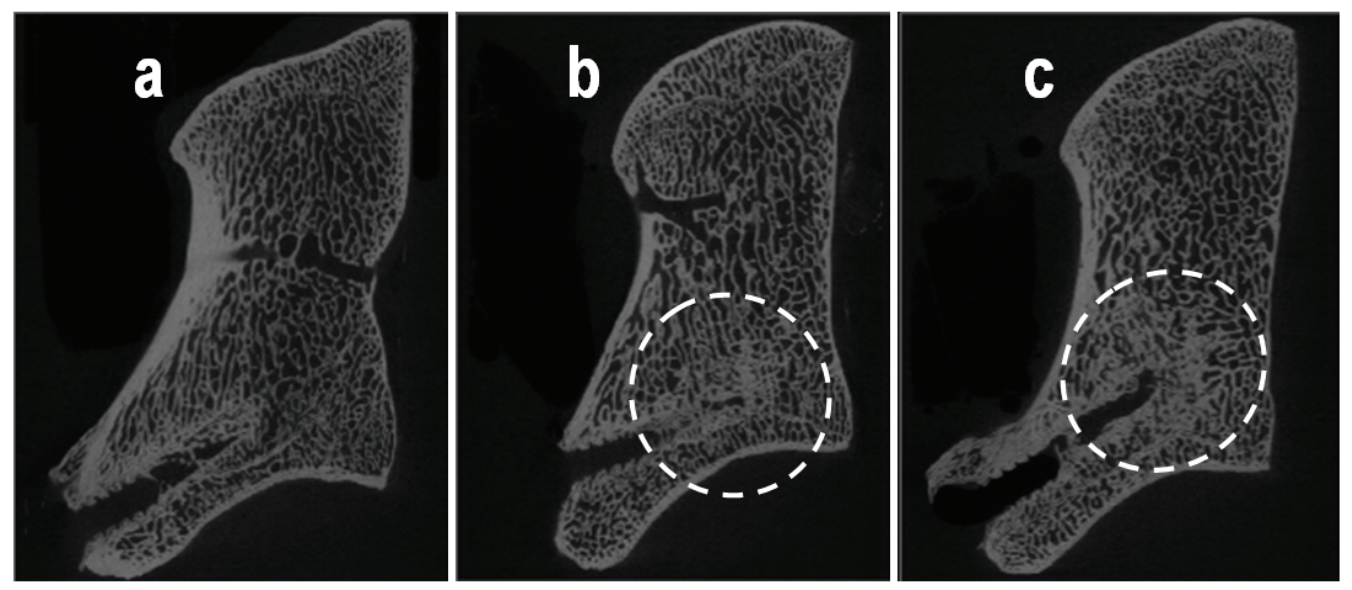


\subsubsection{Histomorphometry}

Exact percentages of new bone formation, old remaining bone matrix, granulation tissue and total bone are reported in Table 4 and Figure 9.

Table 4. Histomorphometrical measurements of old bone, new bone, total bone and granulation tissue (in percentage of total area measured).

\begin{tabular}{|c|c|c|c|c|}
\hline Group & Old bone matrix & New bone matrix & Total bone matrix & Granulation tissue \\
\hline Matrix alone & $22.4 \pm 3.4$ & $19.6 \pm 8.6$ & $42.0 \pm 8.0$ & $58.0 \pm 8.0$ \\
\hline Low & $15.3 \pm 2.3$ & $26.3 \pm 5.5$ & $41.9 \pm 5.2$ & $58.4 \pm 5.2$ \\
\hline Medium & $15.5 \pm 3.5$ & $32.0 \pm 3.6$ & $47.6 \pm 3.6$ & $52.5 \pm 3.6$ \\
\hline High & $9.6 \pm 10.5$ & $27.5 \pm 9.7$ & $37.1 \pm 12.9$ & $62.9 \pm 12.9$ \\
\hline Sham & $34.2 \pm 6.9$ & $7.1 \pm 8.2$ & $41.4 \pm 12.4$ & $58.7 \pm 12.4$ \\
\hline
\end{tabular}

Figure 9. The bars show the mean and SD values of the histomorphometrical measurements of old bone, new bone and granulation tissue (as a percentage of the total area measured). All treatment groups showed significantly higher percentages of new bone than the control group. In contrast, the sham group had the highest percentage of old bone (significant differences compared to the sham group are highlighted with asterisks; the bracket illustrates the significant difference between the matrix alone group and the group with medium TGplPTH $1-34)$.

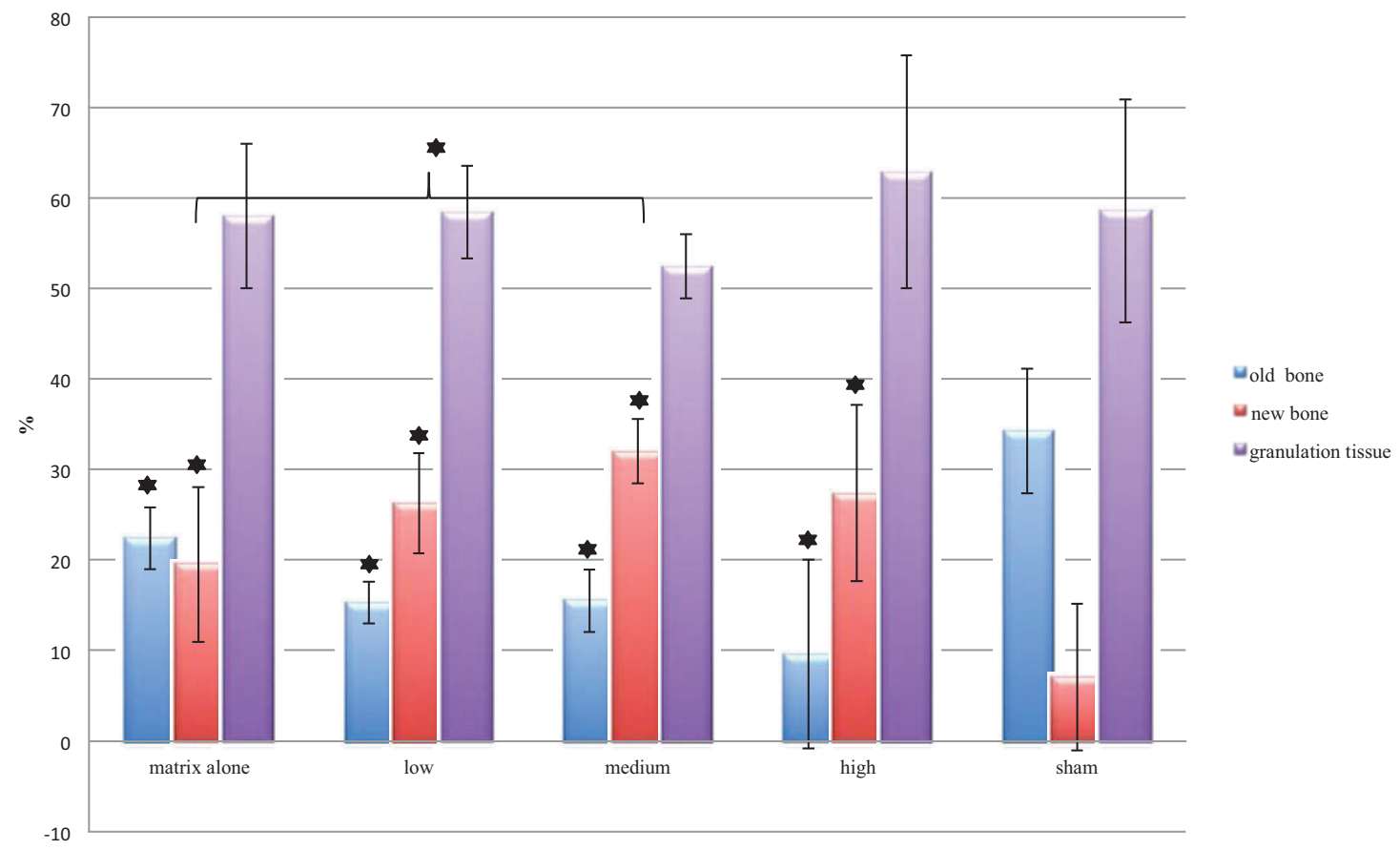

As in the $\mu \mathrm{CTs}$ overall, a high percentage of new bone was found in the augmented region $(19.6 \%-32 \%)$ for all treatment groups, whereas in the sham group, new bone was only detected at the border of the needle path (7.1\%). In all treatment groups, the area adjacent to the tip of the needle seemed to be mainly filled with new bone, partly due to proliferation and partly to an enhanced remodeling and bone formation process (Figure 10). 
Figure 10. Examples of toluidine blue-stained ground sections of all groups; the area at the tip of the needle of all treatment groups showed mainly newly formed bone (indicated by darker blue areas), whereas for the sham group, new bone only was detectable at the border of the needle path. Note the increased trabecular thickness in the augmented area.

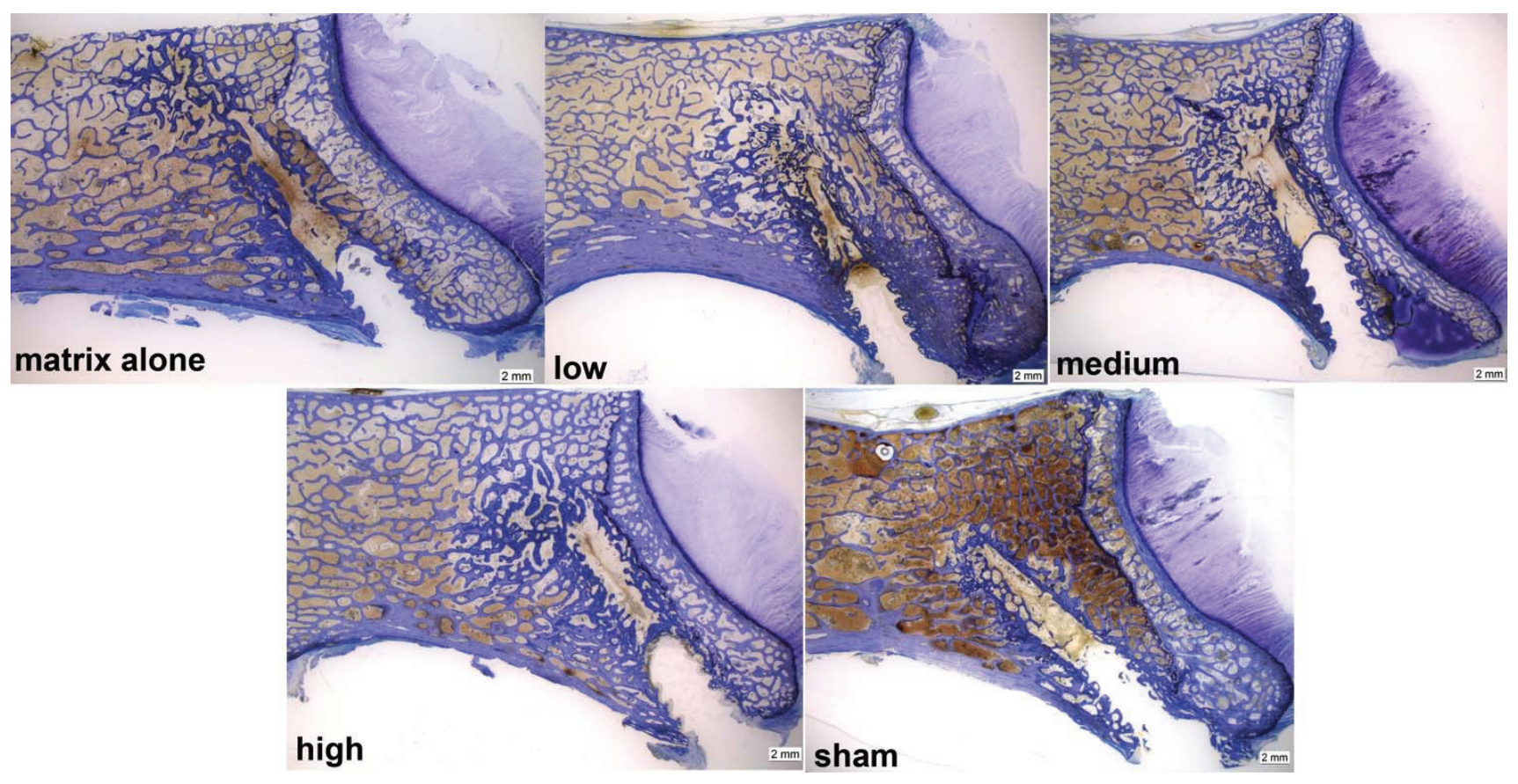

Overall differences between groups in percentage for old and new bone were significant at $p=0.000$. When compared individually, all treatment groups showed significantly higher percentages of new bone than the sham group ( $p$-value ranging between 0.000 and 0.029 ). In contrast, the sham group had the highest percentage of old bone compared to all treatment groups ( $p$-value ranging between 0.000 and 0.009 ). No significant differences between groups were found for the percentages of granulation tissue $(52.4 \%-62.9 \%)$ and of total bone $(37.1 \%-47.56 \%)$. The bone remodeling process was pronounced in all specimens of the treatment groups, but especially for the groups with higher concentrations of $\mathrm{TGplPTH}_{1-34}$. The highest average for new bone (32\%) was found for the group with a medium concentration of $\mathrm{TGplPTH}_{1-34}$. with statistical significant differences also for the group with matrix alone (19.6\%) $(p=0.042)$ (Figure 9).

\subsubsection{Fluorescence}

Means and SD from semi-quantitatively scoring fluorescence sections are reported in Table 5. No fluorescent dye (tetracycline) was detected at 16 weeks, so that it was left out of the table.

The fluorescence images of all treatment groups demonstrated the sphere-like shape distribution of the biomaterial with a red colored area in the middle and a green area towards the periphery. This pattern indicated a radial progression of new bone formation with enhanced bone activity, and precipitation of the fluorescence markers starting in the periphery and the biomaterial being progressively degraded, starting from the periphery towards the middle of the injected region (Figure 11). 
Table 5. Means and SD of the semi-quantitative fluorescence scores for all groups and at different time points show that increased new bone formation occurred mainly at the early time point at six weeks in all PTH derivates compared to controls. At 12 weeks, new bone deposition was lower in all groups, except the matrix alone group.

\begin{tabular}{|c|c|c|}
\hline Group & Calcein green (6w.p.OP) & Xylenol orange (12w.p.OP) \\
\hline Matrix alone & $0.8 \pm 0.4$ & $0.9 \pm 0.9$ \\
\hline Low & $1.0 \pm 0.0$ & $1.1 \pm 0.8$ \\
\hline Medium & $1.5 \pm 0.8$ & $0.8 \pm 0.7$ \\
\hline High & $1.4 \pm 0.5$ & $0.9 \pm 0.9$ \\
\hline Sham & $0.7 \pm 0.5$ & $0.1 \pm 0.3$ \\
\hline
\end{tabular}

Figure 11. This figure shows representative fluorescence images of all groups at the three different time points, as well as one image merged, respectively overlaid together. At six weeks, enhanced bone activity in the injected region could be detected especially for the treatment groups with a medium and high concentration. This localized bone activity decreased at 12 weeks and, with the degradation of the material, finally disappeared at 16 weeks. In the sham group, only the area next to the needle track was enhanced. Note that the area of bone deposition is broader in its diameter in the treated groups compared to the matrix alone.

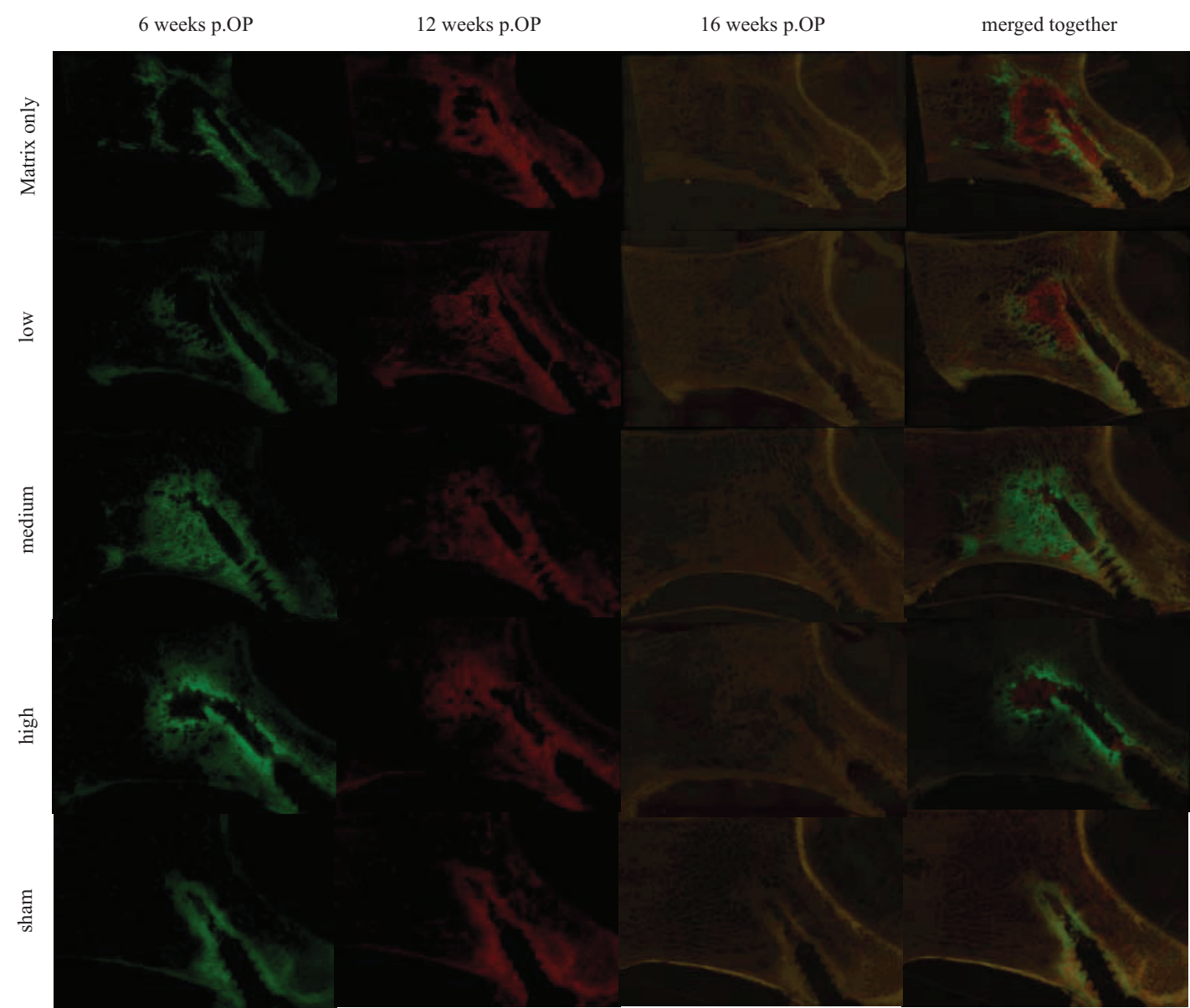


At six weeks, all treatment groups showed enhanced bone activity in the injected area, as indicated by calcein green precipitation. Overall differences between groups in bone activity were significant at $p=0.003$. Especially in the groups with medium and high $\mathrm{TGplPTH}_{1-34}$ concentrations, the bone activity was enhanced with significantly higher values in comparison to the sham group $(p=0.029$ and $p=0.040$ for groups with medium and high concentration, respectively). This localized bone activity decreased at 12 weeks (xylenol orange) and finally disappeared at 16 weeks (tetracycline) proportional to the degradation of the material. For the treatment group with matrix only and for the group with a low concentration of $\mathrm{TGplPTH}_{1-34}$, higher scores were given at 12 weeks versus six weeks, with other treatment groups maintaining stable values during that period. In the sham group, only the area next to the needle track was enhanced for bone activity, which was attributed to the remodeling process of bone debris created by the needle.

\subsubsection{Histologic and Cellular Evaluation}

Means and SD of the semi-quantitative histology scoring are summarized in Table 6.

Table 6. Means and SD of the semi-quantitative histology scoring are given using the average of the three scoring areas for each augmentation site.

\begin{tabular}{|c|c|c|c|c|c|c|c|c|}
\hline Group & $\begin{array}{c}\text { Mononuclea } \\
\text { r cells }\end{array}$ & $\begin{array}{c}\text { Macro- } \\
\text { phages }\end{array}$ & $\begin{array}{c}\text { Multinucleate } \\
\text { d foreign body } \\
\text { cells }\end{array}$ & $\begin{array}{c}\text { Fibro- } \\
\text { blasts }\end{array}$ & $\begin{array}{c}\text { Osteo- } \\
\text { clasts }\end{array}$ & $\begin{array}{c}\text { Amount of } \\
\text { osteoid } \\
\text { seam }\end{array}$ & $\begin{array}{c}\text { Thickness } \\
\text { of osteoid }\end{array}$ & $\begin{array}{c}\text { Residual } \\
\text { material }\end{array}$ \\
\hline $\begin{array}{c}\text { Matrix } \\
\text { alone }\end{array}$ & $1.2 \pm 0.4$ & $1.1 \pm 0.6$ & $0.0 \pm 0.0$ & $0.2 \pm 0.3$ & $0.3 \pm 0.4$ & $0.7 \pm 0.5$ & $0.7 \pm 0.5$ & $0.3 \pm 0.5$ \\
\hline Low & $1.3 \pm 0.3$ & $1.2 \pm 0.5$ & $0.2 \pm 0.2$ & $0.0 \pm 0.0$ & $0.5 \pm 0.3$ & $1.2 \pm 0.6$ & $1.3 \pm 0.6$ & $0.1 \pm 0.1$ \\
\hline Medium & $1.2 \pm 0.4$ & $1.0 \pm 0.2$ & $0.1 \pm 0.2$ & $0.0 \pm 0.0$ & $0.5 \pm 0.4$ & $1.1 \pm 0.7$ & $1.2 \pm 0.7$ & $0.0 \pm 0.0$ \\
\hline High & $1.0 \pm 0.2$ & $0.6 \pm 0.3$ & $0.0 \pm 0.0$ & $0.0 \pm 0.0$ & $0.5 \pm 0.4$ & $1.2 \pm 0.4$ & $1.3 \pm 0.3$ & $0.0 \pm 0.0$ \\
\hline Sham & $0.9 \pm 0.8$ & $0.2 \pm 0.4$ & $0.0 \pm 0.0$ & $0.0 \pm 0.0$ & $0.5 \pm 0.4$ & $0.4 \pm 0.3$ & $0.5 \pm 0.3$ & $0.0 \pm 0.0$ \\
\hline
\end{tabular}

The semi-quantitative histology scoring revealed similar values for all groups. Overall differences between groups were only found for scores of macrophages $(p=0.000)$. Due to the degradation process of the biomaterial, the number of macrophages was significantly higher in $3 / 4$ treatment groups compared to the sham group (the $p$-value was $0.003,0.001$ and 0.009 for groups with matrix only, low and medium concentrations, respectively). However, the low scores of all treatment groups in comparison to the sham group for mononuclear cells and multinucleated foreign body cells indicated an excellent biocompatibility for all formulations. Thick osteoid seams and activated round-shaped osteoblasts evidenced an improved bone activity with higher scores found for all material groups containing $\mathrm{TGplPTH}_{1-34}$ (Figure 12). Residual biomaterial was found only for the matrix only and low concentration groups. 
Figure 12. This figure shows a thin section with many macrophages $(\rightarrow)$ and a thick osteoid seam with activated round-shaped osteoblasts $(\rightarrow)$.

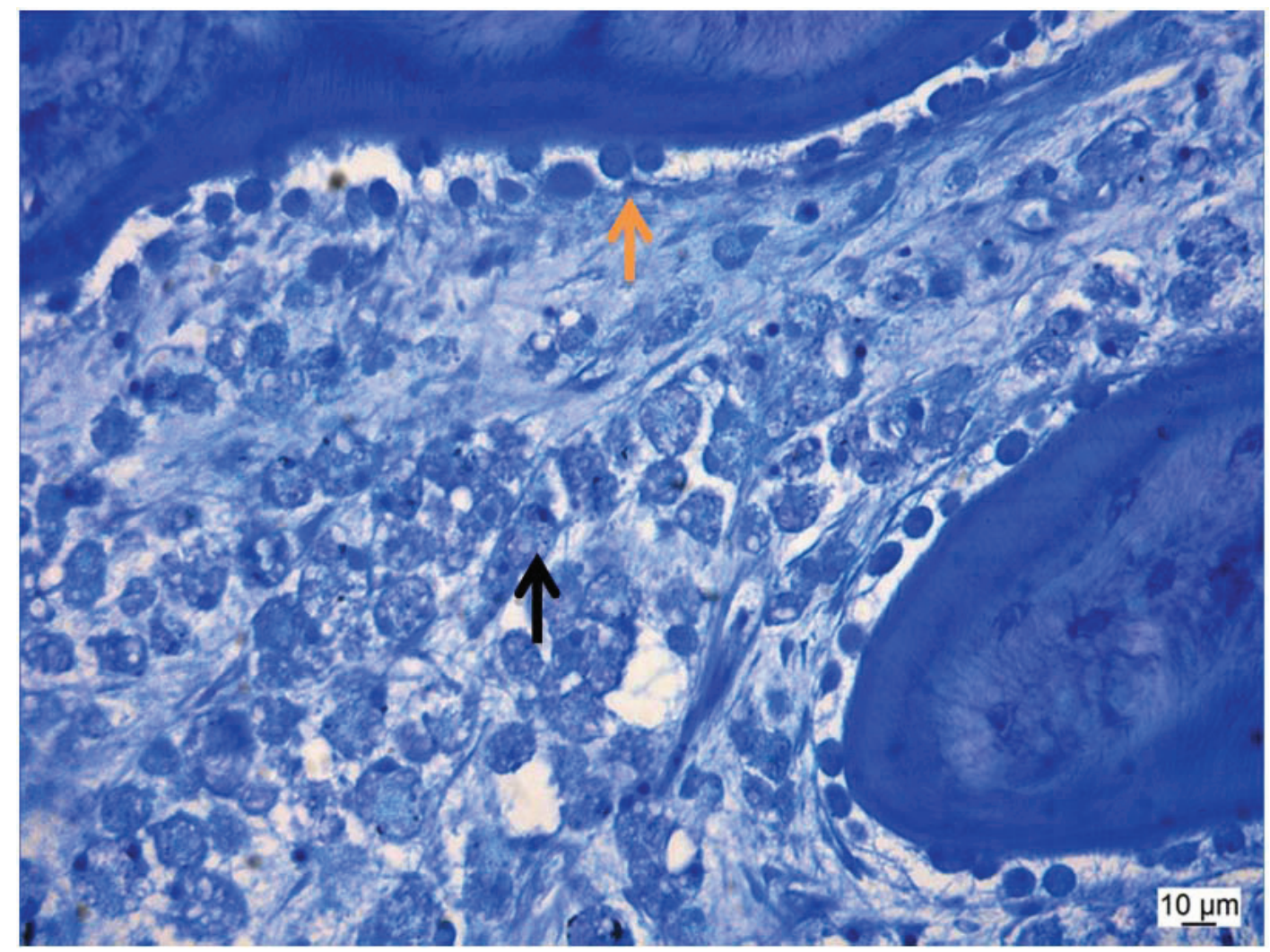

\section{Discussion}

In this study, a novel animal model in sheep was successfully established by using the cervical vertebrae of 17 sheep for the augmentation of cancellous bone with a biomimetic agent. The surgical and evaluation methods proved to be well standardized and highly reproducible. In addition, the efficacy of locally-delivered $\mathrm{TGplPTH}_{1-34}$ incorporated in a fibrin matrix containing strontium carbonate was demonstrated to be a potentially suitable augmentation material for the cervical vertebra.

The surgical method was straightforward and easy to apply, with the aid of the specially developed aiming device. Using fluoroscopic imaging during the surgical procedure, combined with the various analyses, such as $\mu \mathrm{CT}$, histomorphometry and sequential fluorescence dye staining, we were able to demonstrate that drilling and subsequent needle placement was accurate and reproducible. Consequently, we were able to inject test materials in 43/45 augmentation sites into the intertrabecular spacing of cervical vertebral bodies again with accuracy and reproducibility. However, as already discussed in a previous study concerning the tibia and femur augmentation in sheep [20], the trabecular bone density, as well as the trabecular thickness is much higher in sheep than in humans, such that the needle placement and material injection is more difficult to achieve in comparison to humans. In osteoporotic, brittle human bone, the injection needle can be placed in position using manual pressure only, whereas in sheep, a hole had to be drilled with a burr first in order to be able to push the needle forward into the healthy and dense trabecular bone. However, in contrast to the tibia and femur augmentation study, where a mallet had to be used after drilling to push the needle forward into the bone, this was not required for cervical bone augmentation. Therefore, the frequently observed surgical complications in 
the previous study, where the rather frail needle broke during the extraction procedure, were significantly less frequent in the current study (1/45). The harvesting method of the treated vertebrae was easy, fast and highly reproducible, with no need for an additional aiming device to define the region of interest as needed in the previous study [20].

Cardiovascular emboli are noticed in human patients after vertebral augmentation, even with a reported mortality rate of $2 \%$. Emboli were also reported in our previously established femoral and tibia augmentation model [20]. Nevertheless, this cervical vertebral model presented more challenges regarding cardiovascular complications in comparison. Although the same material was used in both studies, the cervical augmentation model revealed a relatively high rate of pulmonary emboli, with two animals (11.76\%) dying immediately on the table with severe signs of PE and five animals (29.4\%) still showing mild signs of PE. In the femoral and tibia augmentation model, a higher leakage of material into the vessels was observed. This may be attributed to the different anatomical location, but probably also to the higher volume of material injected in the femoral and tibia augmentation model (3.5 mL per augmentation site). In human patients, reported emboli are less, but then, they are not routinely followed by lung $\mathrm{CT}$ after augmentation and, therefore, may often go unnoticed as long as no life threatening complication jeopardizes the patient's life. The higher mortality rate in sheep compared to humans may be related to the fact that injection of a relatively high material volume was performed in healthy cancellous bone of the vertebral body and that the sheep have denser bone compared to humans. Nevertheless, the cervical augmentation model presented the valuable opportunity to study the development of PE and its consequences, as they also occur in humans during vertebroplasty [32].

Mechanical testing of the vertebral bodies was not performed. Apart from technical problems to develop an adequate testing system with highly standardized cubes, it also was not of high priority in this study. One of the main reasons was that we did not treat osteoporotic sheep, since there is no adequate large animal model for this disease. Although we assumed that new bone formation with highly active osteoblast activity on new osteoid seams would be visible, it was not expected to significantly change trabecular thickness in healthy animals, since the mechanical load on the vertebrae had not changed. Trabecular thickness was only measured in $\mu \mathrm{CT}$, where only calcified tissue is measured. However, in the histology sections, the increased trabecular thickness due to osteoid deposition is clearly visible in the ground and thin sections.

The three different concentrations (low, medium, high) of $\mathrm{TGplPTH}_{1-34}$ proved to be suitable for cervical augmentation in sheep and showed indications of enhanced localized bone activity in all groups, especially during the first six weeks after surgery. Micro CT analysis, histomorphometry, as well as the evaluation of fluorescent incorporation indicated an increased bone formation and remodeling activity spatially limited to the injected area. As in the previous study [20], sequential fluorescent dye images indicated a radial propensity for new bone forming gradually over time from the periphery towards the middle of the injected regions. These findings support the hypothesis of Arrighi et al. that cell migration and infiltration into the fibrin matrix activate and release $\mathrm{TGplPTH}_{1-34}$ into the surrounding tissue [19]. Our results further confirm those of Yang et al. showing that locally applied strontium has inherent osteoinductive properties itself [25]. However, combining strontium with high and probably even better medium concentrations of $\mathrm{TGplPTH}_{1-34}$ enhanced the overall proliferative effect that was already previously demonstrated by our group in the femoral and tibia 
augmentation model [20]. The exact mechanism by which this co-effect is exerted is not fully known. However, we hypothesize that the accumulation of extracellular matrix induced by strontium increases the migration and infiltration of cells into the fibrin matrix and may also reduce osteoclast activity via apoptosis, again enhancing bone formation [25]. PTH, on the other hand, has anabolic activities on osteoblasts, increasing remodeling activity, as clearly shown in our previous study [20]. Taken together, bone forming properties are therefore enhanced by both ways: the increased osteoblastic activity through PTH and the increased population of living cells throughout the extracellular matrix population via strontium. Our evaluation methods did not allow distinguishing between increased remodeling activity and true new bone formation, as would be found in true osteoinduction. However, if in our current study, the different groups are compared to each other, it is obvious that the groups treated with $\mathrm{TGplPTH}_{1-34}$ showed considerable increased bone formation compared to the matrix alone group. If the material alone in combination with trabecular microdamage would be the reason for the new bone formation, the matrix alone and with $\mathrm{TGplPTH}_{1-34}$-treated groups would be equal in their response. However, the differences in new bone deposition are clearly visible in the fluorescent sections. Therefore, it is safe to assume that the new bone formation is in response to both remodeling activity after material injection and new bone formation after local application of $\mathrm{TGplPTH}_{1-34}$.

The broad precipitation of fluorescence markers at six weeks post-surgery for the groups with medium and high concentrations of $\mathrm{TGplPTH}_{1-34}$ indicate an enhanced, early and fast bone formation, which could be confirmed by histomorphometry with the highest percentage of new bone formation (32.0\%) found for the medium concentration of $\mathrm{TGplPTH}_{1-34}$, followed by $27.5 \%$ new bone formation found for the high concentration of $\mathrm{TGplPTH}_{1-34}$. In comparison, groups with fibrin matrix only or with low concentration of $\mathrm{TGplPTH}_{1-34}$ revealed less overall bone activity and at a later time point (12 weeks). This seems connected to the findings in the $\mu \mathrm{CT}$ analysis, where low quantities of residual material for the groups without or with a low dose of $\mathrm{TGplPTH}_{1-34}$ could be related to higher amounts of remaining material. We assume that the degradation of these formulations is decelerated and therefore reduces the bone enhancing activity due to the delayed release of activated $\mathrm{TGpIPTH}_{1-34}$. To prove this theory in a future study, an earlier time point for the first fluorescence injection could be taken into consideration in order to confirm that the enhanced bone activity of medium and high concentrations of $\mathrm{TGplPTH}_{1-34}$ shows a faster degradation of material, in correlation with a stronger enhanced bone activity.

In systemic applications of $\mathrm{TGplPTH}_{1-34}$, a great concern is that high PTH concentrations may increase osteoclast activity [33]. This may be reflected by a lower percentage of newly formed bone, in addition to increased bone resorption zones and higher numbers of osteoclasts. Due to the mechanism of PTH, the influence on osteoclasts must be evaluated, when PTH is applied as a local peptide. In this study, no increase of osteoclasts and no resorption zones were found for any of the PTH concentrations. These findings were attributed to the use of the peptide covalently bound to the fibrin matrix that is only released and activated via local cell invasion and enzymatic cleavage. We therefore suggest that the osteoclast-enhancing effect of PTH shown by others depends not only on the higher dose, but mainly on the route of administration, which could be avoided by delivering the peptide locally and covalently bound to the fibrin matrix. 


\section{Conclusion}

The cervical augmentation model in sheep described in this study proved to be a suitable animal model to evaluate the biocompatibility and new bone formation properties of new biomaterials, intended for vertebral bone augmentation. Although the model revealed to be more challenging, due to cardiovascular complications compared to the femoral and tibia augmentation model, this reflected well the human situation. Nevertheless, the results demonstrated that the TGplPTH $\mathrm{TH}_{1-34}$ was safe to use in sheep, since no signs of inflammation were detected and fast biomaterial degradation took place. All treatment groups were more effective than the sham control. Among these, the medium concentration of $\mathrm{TGplPTH}_{1-34}$ induced the best bone enhancing effect. Whether this effect would also be elicited in osteoporotic bone in humans is speculative at this point. Due to the absence of a true osteoporotic large animal model, this could only be verified in a clinical trial study in humans.

\section{Acknowledgments}

The authors thank their industrial partners for financing the study.

\section{Author Contributions}

K.Kl. was the Ph.D. student and involved in all aspects of the study, including writing the manuscript. J.S. was involved in developing the biomaterial. P.W.K. was the anesthetist and was involved in demonstrating embolization. N.F. was the anesthetist and was involved in demonstrating embolization. K.K. performed histology and was involved in developing bone sampling. M.M. was the radiologist and performed all CT including evaluation. T.S. designed the device and was involved in all aspects of the study. B.R. was the head of the experiment, supervisor of the Ph.D. program and was involved in all aspects of the study.

\section{Disclosure}

The study was financed by our industrial partner, Kuros Biosurgery AG, Technoparkstrasse 1, 8005 Zurich, Switzerland.

\section{Conflicts of Interest}

The authors declare no conflict of interest.

\section{References and Notes}

1. Rapado, A. General management of vertebral fractures. Bone 1996, 18, 191S-196S.

2. Old, J.L.; Calvert, M. Vertebral compression fractures in the elderly. Am. Fam. Phys. 2004, 69, 111-116.

3. Lewis, G. Percutaneous vertebroplasty and kyphoplasty for the stand-alone augmentation of osteoporosis-induced vertebral compression fractures: Present status and future directions. J. Biomed. Mater. Res. B Appl. Biomater. 2007, 81, 371-386. 
4. Lavelle, W.; Carl, A.; Lavelle, E.D.; Khaleel, M.A. Vertebroplasty and kyphoplasty. Anesthesiol. Clin. 2007, 25, 913-928.

5. Hulme, P.A.; Krebs, J.; Ferguson, S.J.; Berlemann, U. Vertebroplasty and kyphoplasty: A systematic review of 69 clinical studies. Spine (Phila Pa 1976) 2006, 31, 1983-2001.

6. Predey, T.A.; Sewall, L.E.; Smith, S.J. Percutaneous vertebroplasty: New treatment for vertebral compression fractures. Am. Fam. Phys. 2002, 66, 611-615.

7. Silverman, S.L. The clinical consequences of vertebral compression fracture. Bone 1992, 13 (Suppl 2), S27-S31.

8. Garfin, S.R.; Yuan, H.A.; Reiley, M.A. New technologies in spine: Kyphoplasty and vertebroplasty for the treatment of painful osteoporotic compression fractures. Spine (Phila Pa 1976) 2001, 26, $1511-1515$.

9. Lemke, D.M. Vertebroplasty and kyphoplasty for treatment of painful osteoporotic compression fractures. J. Am. Acad. Nurse Pract. 2005, 17, 268-276.

10. Phillips, F.M.; Todd Wetzel, F.; Lieberman, I.; Campbell-Hupp, M. An in vivo comparison of the potential for extravertebral cement leak after vertebroplasty and kyphoplasty. Spine (Phila Pa 1976) 2002, 27, 2173-2178; discussion 2178-2179.

11. Jensen, M.E.; Evans, A.J.; Mathis, J.M.; Kallmes, D.F.; Cloft, H.J.; Dion, J.E. Percutaneous polymethylmethacrylate vertebroplasty in the treatment of osteoporotic vertebral body compression fractures: Technical aspects. AJNR Am. J. Neuroradiol. 1997, 18, 1897-1904.

12. Cotten, A.; Boutry, N.; Cortet, B.; Assaker, R.; Demondion, X.; Leblond, D.; Chastanet, P.; Duquesnoy, B.; Deramond, H. Percutaneous vertebroplasty: State of the art. Radiographics 1998, 18, 311-320; discussion 320-323.

13. Frankel, B.M.; Monroe, T.; Wang, C. Percutaneous vertebral augmentation: An elevation in adjacent-level fracture risk in kyphoplasty as compared with vertebroplasty. Spine J. 2007, 7, 575-582.

14. Nardi, A.; Tarantino, U.; Ventura, L.; Armotti, P.; Resmini, G.; Cozzi, L.; Tonini, G.; Ramazzina, E.; Rossini, M. Domino effect: Mechanic factors role. Clin. Cases Miner. Bone Metab. 2011, 8, 38-42.

15. Poole, K.E.; Reeve, J. Parathyroid hormone-A bone anabolic and catabolic agent. Curr. Opin. Pharmacol. 2005, 5, 612-617.

16. Carter, P.H.; Schipani, E. The roles of parathyroid hormone and calcitonin in bone remodeling: Prospects for novel therapeutics. Endocr. Metab. Immune Disord. Drug Targets 2006, 6, 59-76.

17. Pfeilschifter, J.; Laukhuf, F.; Muller-Beckmann, B.; Blum, W.F.; Pfister, T.; Ziegler, R. Parathyroid hormone increases the concentration of insulin-like growth factor-i and transforming growth factor beta 1 in rat bone. J. Clin. Investig. 1995, 96, 767-774.

18. Whitfield, J.F. Osteoporosis-treating parathyroid hormone peptides: What are they? What do they do? How might they do it? Curr. Opin. Investig. Drugs 2006, 7, 349-359.

19. Arrighi, I.; Mark, S.; Alvisi, M.; von Rechenberg, B.; Hubbell, J.A.; Schense, J.C. Bone healing induced by local delivery of an engineered parathyroid hormone prodrug. Biomaterials 2009, 30, $1763-1771$. 
20. Klein, K.; Zamparo, E.; Kronen, P.W.; Kampf, K.; Makara, M.; Steffen, T.; von Rechenberg, B. Bone augmentation for cancellous bone-Development of a new animal model. BMC Musculoskelet. Disord. 2013, 14, 200.

21. Ammann, P. Strontium ranelate: A physiological approach for an improved bone quality. Bone 2006, 38, 15-18.

22. Marie, P.J. Strontium ranelate: A physiological approach for optimizing bone formation and resorption. Bone 2006, 38, S10-S14.

23. Brown, E.M. Is the calcium receptor a molecular target for the actions of strontium on bone? Osteoporos. Int. 2003, 14 (Suppl 3), S25-S34.

24. Hurtel-Lemaire, A.S.; Mentaverri, R.; Caudrillier, A.; Cournarie, F.; Wattel, A.; Kamel, S.; Terwilliger, E.F.; Brown, E.M.; Brazier, M. The calcium-sensing receptor is involved in strontium ranelate-induced osteoclast apoptosis. New insights into the associated signaling pathways. J. Biol. Chem. 2009, 284, 575-584.

25. Yang, F.; Yang, D.; Tu, J.; Zheng, Q.; Cai, L.; Wang, L. Strontium enhances osteogenic differentiation of mesenchymal stem cells and in vivo bone formation by activating wnt/catenin signaling. Stem Cells 2011, 29, 981-991.

26. Benneker, L.M.; Gisep, A.; Krebs, J.; Boger, A.; Heini, P.F.; Boner, V. Development of an in vivo experimental model for percutaneous vertebroplasty in sheep. Vet. Comp. Orthop. Traumatol. 2012, 25, 173-177.

27. Krebs, J.; Ferguson, S.J.; Hoerstrup, S.P.; Goss, B.G.; Haeberli, A.; Aebli, N. Influence of bone marrow fat embolism on coagulation activation in an ovine model of vertebroplasty. J. Bone Joint Surg. Am. 2008, 90, 349-356.

28. Krebs, J.; Ferguson, S.J.; Nuss, K.; Leskosek, B.; Hoerstrup, S.P.; Goss, B.G.; Shaw, S.; Aebli, N. Plasma levels of endothelin-1 after a pulmonary embolism of bone marrow fat. Acta Anaesthesiol. Scand. 2007, 51, 1107-1114.

29. Aebli, N.; Krebs, J.; Schwenke, D.; Davis, G.; Theis, J.C. Cardiovascular changes during multiple vertebroplasty with and without vent-hole: An experimental study in sheep. Spine (Phila Pa 1976) 2003, 28, 1504-1511; discussion 1511-1512.

30. Boger, A.; Benneker, L.M.; Krebs, J.; Boner, V.; Heini, P.F.; Gisep, A. The effect of pulsed jet lavage in vertebroplasty on injection forces of pmma bone cement: An animal study. Eur. Spine J. 2009, 18, 1957-1962.

31. Measurements are not in the content of this paper and will be published elsewhere.

32. Data not shown here.

33. Robling, A.G.; Kedlaya, R.; Ellis, S.N.; Childress, P.J.; Bidwell, J.P.; Bellido, T.; Turner, C.H. Anabolic and catabolic regimens of human parathyroid hormone 1-34 elicit bone- and envelope-specific attenuation of skeletal effects in sost-deficient mice. Endocrinology 2011, 152, 2963-2975.

(C) 2014 by the authors; licensee MDPI, Basel, Switzerland. This article is an open access article distributed under the terms and conditions of the Creative Commons Attribution license (http://creativecommons.org/licenses/by/3.0/). 